\title{
Is fresh, leucodepleted, whole blood transfusion superior to blood component transfusion in pediatric patients undergoing spinal deformity surgeries? A prospective, randomized study analyzing postoperative serological parameters and clinical recovery
}

\author{
P. Keerthi Vasan ${ }^{1} \cdot$ S. Rajasekaran ${ }^{2}$ (D) Vibhu Krishnan Viswanathan ${ }^{1} \cdot$ Ajoy Prasad Shetty ${ }^{1} \cdot$ Rishi Mugesh Kanna $^{1}$
}

Received: 11 June 2020 / Revised: 30 January 2021 / Accepted: 24 February 2021 / Published online: 16 March 2021

(c) The Author(s), under exclusive licence to Springer-Verlag GmbH Germany, part of Springer Nature 2021

\begin{abstract}
Purpose To compare the effectiveness of fresh whole blood (FWB) and blood component transfusion in improving clinical outcome and serological parameters in the early postoperative period following spinal deformity surgery.

Methods Patients undergoing major spinal deformity surgeries involving $\geq 6$ levels of fusion and expected blood loss $\geq 750 \mathrm{ml}$ between September 2017 and August 2018 were included in the study. The patients were randomized into two groups: FWBG and CG, receiving fresh whole blood and component transfusions, respectively.

Results A total of 65 patients with spinal deformities of different etiologies were included. The mean age was 14.0 and 14.9 years in FWB and CG, respectively. All other preoperative parameters were comparable. The mean fusion levels and surgical time were 11.1 and $221.20 \mathrm{~min}$ in FWB, as compared with 10.70 and 208.74 minutes in CG, respectively. Intraoperative blood losses were $929 \mathrm{ml}$ (FWBG) and $847 \mathrm{ml}(\mathrm{CG})$, and the mean volumes of transfusion were 1.90 (FWBG) and 1.65 units (CG). FWBG was significantly superior to $\mathrm{CG}$ in the following clinical and laboratory parameters: duration of oxygen dependence [36.43 (FWBG) vs. 43.45 h (CG); $P=0.0256]$, mean arterial pH [7.442 (FWBG) vs. 7.394 (CG); $p<0.001]$, interleukin-6 [30.04 (FWBG) vs. 35.10 (CG); $p<0.019$ ], mean duration of HDU stay [40.6 hours (FWBG) vs 46.51 hours $(\mathrm{CG}) ; p=0.0234]$ and postoperative facial puffiness [7/30 in FWBG vs. 18/35 (CG) $(P<0.02)]$.

Conclusion FWB transfusion can potentially improve the immediate postoperative outcome in patients undergoing major spinal deformity surgeries by reducing the duration of intensive care unit stay and oxygen dependence. The other potential benefits of this practice, based on our study, include a reduced inflammatory response (reduced lactate and IL-6) and postoperative facial puffiness. However, further large-scale validation studies in future are necessary to precisely determine the role of FWB in spine surgeries.

Level of evidence II Diagnostic: individual cross-sectional studies with the consistently applied reference standard and blinding.
\end{abstract}

Keywords Blood transfusion - Spine deformity surgery · Fresh whole blood transfusion · Component transfusion ·

Postoperative clinical recovery

S. Rajasekaran

rajasekaran.orth@gmail.com

1 Department of Spine Surgery, Ganga Medical Centre and Hospital, 313, Mettupalayam Road, Sai Baba Colony, Coimbatore, Tamil Nadu 641001, India

2 Department of Orthopaedics and Spine Surgery, Ganga Medical Centre and Hospital, 313, Mettupalayam Road, Sai Baba Colony, Coimbatore, Tamil Nadu 641001, India

\section{Introduction}

Major spinal deformity surgeries (SDS) have high morbidity owing to a high incidence of associated intraoperative (IO) and postoperative (PO) complications [1]. Early postoperative rehabilitation plays a pivotal role in improving the overall outcomes of these patients. Complex surgical procedures involve longer operating time, and the inevitable major blood losses associated with these surgeries demand multiple transfusions [2, 3]. The existing literature has profound 
data regarding preoperative optimization, blood loss prevention, and optimum transfusion criteria in major SDS [4].

The concepts of blood transfusion and banking originated during World War (WW)-I and further expanded during WW-II [5]. Although whole blood (WB) was the preferred primary resuscitation fluid initially [5], with our enhanced ability to segregate different components over the following decades, transfusion of individual blood components (CT - component transfusion) became the predominant transfusion approach globally $[1,6,7]$. However, over the past decade, there has been a resurgence in the use of WB in its fresh or modified forms in cardiothoracic surgeries, transplantation, and post-traumatic transfusions [5, 8-10]. In major SDS involving large volumes of transfusion, CT is still the widely implemented practice [1]. With the background of rapidly evolving shifts in the paradigm of transfusion medicine and progressively increasing numbers of complex spine surgeries (CSS) worldwide, it may be "need of the hour" from a spine surgeon's perspective to determine the ideal transfusate for such patients. The current prospective randomized-controlled trial (RCT) was thus planned to analyze and compare the effects of fresh WB and CT on postoperative laboratory parameters and clinical recovery of patients undergoing major SDS. To our knowledge, there is no such comparative study involving patients undergoing elective CSS till date.

\section{Methods}

Study design: This prospective RCT was conducted in a tertiary spine center between September 2017 and August 2018 after approval from Institutional review board (IRB). Written informed consent was obtained from patients and their legal guardians before enrolment.

Study participants: Patients $\leq 18$ years of age undergoing corrective surgery for scoliosis and kyphosis involving vertebral fusion $\geq 6$ levels and expected blood loss (BL) $\geq 750 \mathrm{ml}$ were included. Patients with cardiac comorbidities, blood dyscrasias, and history of previous transfusion reactions were excluded. Preoperatively, the participants were assigned to either fresh whole blood (FWBG) or component group (CG) depending on randomization chart prepared using a software program (www.randomizer.org).

Intraoperative protocol: Techniques to reduce IOBL including tranexamic acid infusion (1 $\mathrm{g}$ at induction, followed by $1 \mathrm{~g}$ at 3-hourly intervals, up to $3 \mathrm{~g}$ dose), proper patient positioning, surgical site infiltration with lignocaine/ adrenaline mixture and maintaining appropriate IO mean arterial pressure (MAP) (of $\geq 65 \mathrm{mmHg}$ during exposure and around $85 \mathrm{mmHg}$ during crucial steps including instrumentation, decompression and deformity correction [11]) were uniformly followed in all patients.
Procurement of blood and processing: All blood products were acquired from a random donor pool and utilized after stringent screening for infections in an in-house Government-approved blood bank facility. Chemo-immuno analysis (Abbott-Architect plus-i1000SR) was utilized for screening for HIV, HBsAg, HCV, and syphilis, while rapid-card test was used for malarial parasites. Blood bag CPD/SAGM-2 with inline leukocyte filter for WB (TERUMO PENPOL) was used for blood collection from donors. Leuco-reduction was then carried out by an inline leuco-filter with gravity-assisted transfer at $90 \mathrm{~cm}$ height. The second collection bag contained $63 \mathrm{ml}$ of CPD solution (citric acid monohydrate, sodium citrate dehydrate, sodium dihydrogen phosphate dehydrate, and dextrose anhydrous) as anticoagulant. Fresh WB was utilized within $24 \mathrm{~h}$ of collection. Components were separated by centrifugation and stored in a bag containing SAGM-2 (sodium chloride, adenine, dextrose, and mannitol). Leucoreduction was done in both groups, which has proven benefits including a. prevention of human leukocyte antigens (HLA) and human platelet antigens (HPA) immunization, b. reduction of platelet refractoriness, and c. prevention of CMV/ EBV/HTLV infections [12]. Leuco-reduction is also effective in reducing febrile non-hemolytic transfusion reactions (FNHTR). The separated packed red blood cells (PRBCs) were stored at $1-6{ }^{\circ} \mathrm{C}$, fresh frozen plasma (FFP) at $\leq-18^{\circ} \mathrm{C}$ and platelets under continual agitation at 20 to $20^{\circ} \mathrm{C}$.

Administration of blood products: The decision regarding the need and volume of blood/ blood product replenishment was taken based on the volume of blood loss, preoperative hematocrit and intraoperative/ postoperative hematocrit values. In the $\mathrm{CG}$, the components were always transfused in an equivalent ratio of 1:1:1 (packed RBC:fresh frozen plasma:platelets). The safety of such a "balanced" approach for component transfusion (also known as "damage control resuscitation") has been previously described in trauma situations.

Postoperative protocol: Routinely, all patients undergoing major SDS at our hospital are shifted to a high dependency unit (HDU) postoperatively. The strict criteria for shifting outpatients from HDU to the regular bed include visual analog scale for pain (VAS) $\leq 4$, hemoglobin $(\mathrm{Hb}) \geq 8 \mathrm{~g} \%$, tolerating liquids orally, adequate urine output $(>1 \mathrm{ml} / \mathrm{kg} /$ $\mathrm{hr}$ ), and maintaining oxygen saturation $(\mathrm{SpO} 2) \geq 93 \%$ on room air. All patients were started on intravenous fluids on postoperative days (POD) 0 and commenced on oral fluids on POD1 after the return of bowel sounds. Further on, oral diet was gradually recommenced based on their tolerability on POD2/3. All patients were also encouraged to sit up or stand on POD1 and ambulated based on their pain tolerance and analgesic dosage on POD2/3. According to our protocol, all patients get discharged around POD 7 to 9 , based on their recovery and wound status. Most of our patients travel from long distances, and therefore we usually do not plan earlier discharge from hospital following major SDS. 
Data collection: Preoperatively, demographic data, diagnosis, comorbidities, facial measurements (Laskin's method) [13], blood group/ Rh type of recruited patients were recorded. IO details, including fusion levels, graft type, duration of procedure, blood loss (suction apparatus, gauze/ pad weight), volume of transfusion, and complications were noted.

During the postoperative period, details regarding overall BL via the surgical drain, 6-hourly vitals monitoring [(heart rate (HR), respiratory rate (RR), $\mathrm{SpO} 2$ and temperature (temp)], inotrope requirement, duration of ventilator/ oxygen support, postoperative transfusion, length of HDU stay, and complications like transfusion reactions, facial puffiness, respiratory difficulty, and paralytic ileus were recorded.

Assessment of clinical recovery and laboratory parameters: Subjective and objective well-being scores were evaluated for the first 3 PODs using Likert scale [14]. Assessment of complete blood counts (CBC), coagulation profile, serum electrolytes, renal function and arterial blood gas $(\mathrm{ABG})$ analysis was also performed in all patients preoperatively and daily for first 3 PODs. Additionally, serum calcium profile, lactate and interleukin-6 (IL-6) levels were obtained at the end of 72 postoperative hours. The duration of postoperative hospital stay and return to preoperative activity levels were also compared.

Statistical analysis: Descriptive analysis was performed for analyzing the distribution of demographic variables like age, gender, and weight. Intraoperative and postoperative variables (including biochemical parameters) were assessed statistically using t- and chi-square tests for continuous, categorical variables. Statistical significance was defined by $p \leq 0.05$.

\section{Results}

Demographic profile of patients: Sixty-five patients were recruited for this study and randomized into FWBG (30 patients) and CG (35 patients). All patients received only their assigned blood products during and after surgery. The mean age of patients was $14.0 \pm 3.07$ years and $14.9 \pm 2.67$ years in FWBG and CG, respectively. The mean body weight was $28.83 \mathrm{~kg}$ and $32.37 \mathrm{~kg}$ in FWBG and CG, respectively. The demographic characteristics of patients belonging to both groups were similar (Table 1). The etiology of deformities included adolescent idiopathic scoliosis, early onset scoliosis, neuromuscular scoliosis and neurofibromatosis in 31(14 FWBG \& $17 \mathrm{CG}$ ), 16 (6 FWBG \& $10 \mathrm{CG}), 15$ (8 FWBG \& $7 \mathrm{CG}$ ) and $3(2 \mathrm{FWBG} \& 1 \mathrm{CG})$ patients, respectively.

Intraoperative details: The mean fusion levels were $11.10 \pm 1.98$ and $10.70 \pm 2.56$ in FWBG and CG, respectively. The duration of procedure and IOBL
Table 1 Baseline characteristics of patients [Fresh Whole Blood group (FWBG) vs Component group (CG)]

\begin{tabular}{llll}
\hline & FWBG $(30)$ & CG $(35)$ & $p$ value \\
\hline Age (Years) & $14.0( \pm 3.07)$ & $14.9( \pm 2.67)$ & 0.104 \\
Sex (F:M) & $23: 7$ & $29: 6$ & 0.534 \\
Weight (kg) & $28.83( \pm 6.53)$ & $32.37( \pm 11.17)$ & 0.119 \\
Adolescent Idiopathic & 14 & 17 & \\
$\quad$ Scoliosis (AIS) & & & \\
Early Onset Scoliosis & 6 & 10 & \\
Neuromuscular Scoliosis & 8 & 7 & \\
Neurofibromatosis & 2 & 1 & \\
\hline
\end{tabular}

were $221.20 \pm 58.20 \mathrm{~min}$ and $929 \pm 470.05 \mathrm{ml}$ in FWBG, respectively; as against $208.74 \pm 59.46 \mathrm{~min}$ and $847.14 \pm 451.29 \mathrm{ml}$ in CG. The mean intraoperative transfusion performed in the FWBG was 1.90 units compared to 1.65 units of reconstituted components in CG. There was no statistically significant difference in any of these intraoperative variables between the groups (Table 2).

Postoperative details: The duration of HDU stay was significantly lower in $F W B G$, as compared with $C G$ $(40.6 \pm 9.20 \mathrm{~h}$ vs. $46.51 \pm 11.02 \mathrm{~h})$. The duration of oxygen dependence (i.e., need for administration of some form of oxygen) was also significantly lower in FWBG than $C G(36.43 \pm 9.88 h v s .43 .45 \pm 14.21 h)$. The amount of postoperative blood loss via drain was greater in FWBG ( $516.84 \mathrm{ml}$ vs. $406.19 \mathrm{ml}$ ), although this difference was not statistically significant ( $p=0.053)$. During the first $72 \mathrm{~h}$, there were no significant differences in the vital parameters between the two groups (even after matching patients, based on their blood losses) (Table 3).

Clinical recovery parameters: The subjective and objective well-being scores were statistically similar in the two groups. Eighteen and 7 patients in $C G$ and $F W B G$, respectively, developed significant facial puffiness postoperatively. This difference was statistically significant $(p=0.02)$. This increase in facial puffiness was most notable in both the groups on PODs 2 and 3 .

Table 2 Intraoperative parameters [Fresh Whole Blood group (FWBG) vs Component group (CG)]

\begin{tabular}{lcll}
\hline & FWBG $(30)$ & CG $(35)$ & $p$ value \\
\hline $\begin{array}{l}\text { Mean levels of fusion } \\
\begin{array}{c}\text { Duration of proce- } \\
\text { dure (mins) }\end{array}\end{array}$ & $11.10( \pm 1.98)$ & $10.70( \pm 2.56)$ & 0.493 \\
$\begin{array}{c}\text { Intraoperative blood } \\
\text { loss (ml) }\end{array}$ & $929( \pm 470.05)$ & $847.14( \pm 451.29)$ & 0.477 \\
$\begin{array}{l}\text { Intraoperative trans- } \\
\text { fusion }\end{array}$ & & & \\
\begin{tabular}{l} 
(units) \\
\hline
\end{tabular} & $1.90( \pm 0.712)$ & $1.65( \pm 0.774)$ & 0.181 \\
\hline
\end{tabular}


Table 3 Postoperative parameters [Fresh Whole Blood group (FWBG) vs Component group $(\mathrm{CG})]$

\begin{tabular}{llll}
\hline & FWBG (30) & CG (35) & $p$ value \\
\hline Mean HR (per min) & $90.97(67-112)$ & $89.77(66-110)$ & 0.672 \\
Mean oxygen saturation (in \%) & $99(97-100)$ & $99(98-100)$ & 0.677 \\
Mean RR (per min) & $20.90(18-28)$ & $20.14(16-26)$ & 0.225 \\
Post-op drain collection (in ml) & $516.84(270-550)$ & $406.19(170-500)$ & 0.053 \\
Duration of HDU stay (in h) & $40.6( \pm 9.20)$ & $46.51( \pm 11.02)$ & 0.0234 \\
Duration of O2 dependence & & & \\
(in h) & $36.43( \pm 9.88)$ & $43.45( \pm 14.21)$ & 0.0256 \\
\hline
\end{tabular}

It was also observed that in patients with postoperative facial puffiness, serum IL-6 was also significantly elevated $(p<0.001)$. There was no significant difference in pain scores (mean VAS $3.0 \pm 0.8$ in FWBG vs. $2.9 \pm 0.6$ in CG) or mean time to ambulation (mean time to ambulation $55.6 \pm 12.7$ in FWBG vs. $56.5 \pm 7.6$ in $\mathrm{CG}$ ).

Laboratory parameters: There was a statistically significant difference in the mean arterial $\mathrm{pH}$ between the two groups, with $C G$ patients having a more acidic mean $\mathrm{pH}$ of 7.394 than 7.442 in FWBG $(p<0.001)$. Serum IL-6 level was also significantly higher in $C G(35.10 \mathrm{pg} / \mathrm{ml})$, as compared with FWBG $(30.04 \mathrm{pg} / \mathrm{ml})(p=0.019)$. The serum calcium was marginally higher $(9.68 \mathrm{mg} / \mathrm{dl} v \mathrm{~s} .9 .28 \mathrm{mg} /$ dl) in FWBG, which was also statistically significant. PT, postoperative $\mathrm{Hb}$, and serum electrolytes were similar in the two groups. The total leucocyte counts were also similar between the two groups preoperatively and postoperatively [CG: $11.03 \pm 3.3 * 10^{3}$ (pre-op) vs $15.1 \pm 21.8$ $* 10^{3}$ (first post-op day); FWBG: $* 10^{3}$ (pre-op) vs. $* 10^{3}$

Table 4 Biochemical parameters

\begin{tabular}{lllll}
\hline & GROUP & Mean & Std. Deviation & $p$ value \\
\hline $\begin{array}{l}\text { Prothrombin time } \\
\text { (secs) }\end{array}$ & CG & 14.4857 & .84510 & 0.175 \\
& & & & \\
pH & FWBG & 14.2014 & .80075 & \\
& CG & 7.39486 & .053878 & $<\mathbf{0 . 0 0 1}$ \\
Sodium (mEq/L) & FWBG & 7.44250 & .037834 & \\
& CG & 137.371 & 2.4625 & \\
Potassium (mEq/L) & FWBG & 136.710 & 3.7828 & 0.4 \\
& FWBG & 3.9300 & .44341 & \\
Chloride (mEq/L) & CG & 100.74 & 2.466 & \\
& FWBG & 100.23 & 3.848 & 0.581 \\
Lactate (mg/dl) & CG & 1.490 & .4396 & \\
& FWBG & 1.417 & .4950 & 0.532 \\
Calcium (mg/dl) & CG & 9.286 & .5419 & \\
& FWBG & 9.680 & .5499 & $\mathbf{0 . 0 0 5}$ \\
Interleukin-6 & & & & \\
(pg/ml) & CG & 35.1043 & 9.22473 & \\
& FWBG & 30.0477 & 7.46088 & $\mathbf{0 . 0 1 9}$ \\
\hline
\end{tabular}

(first post-op day); $p>0.05]$. Despite statistically significant difference in the duration of HDU stay, there was no statistically significant difference in the duration of postoperative hospital stay (overall mean length of hospital stay $8.2 \pm 1.3$ days) and incidence of postoperative complications between the groups (Table 4).

\section{Discussion}

The concepts regarding the approach to transfusion have continually changed since early twentieth century [5, 7-9]. The transfusion practice has evolved from initial use of WB and modified WB only to the current, universal use of CT for volume replenishment $[15,16]$. This approach involving the predominant use of CT has stemmed from the purported benefits like better resource utilization, longer components' shelf-life, and greater blood product safety $[8,17]$. Nevertheless, there are no clear scientific reasons to explain the clinical advantages of components over WB; and the current recommendations are based upon expert opinions, past data from modified WB (which was used during a different era of transfusion medicine) and experiments in euvolemic (rather than volume-depleted) patients [10]. Recently, critical views have been put forth regarding the adverse effects of long-term storage of blood components (BC), including its adverse effects on inflammation, immune function, and decreased ability to deliver oxygen to microvasculature [5, 7-9]. There are also no prospective studies to evaluate the clinical impact of transfusing such long-stored BC to critically-ill or surgical patients[10].

In the field of emergency trauma, the potential drawbacks of stored BC transfusion like dilutional coagulopathy, acidosis, dyselectrolytemia and hypothermia have been recognized; and "balanced" or "hemostatic" resuscitation approach is gaining wide acceptance. This "damage control resuscitation" (DCR) involves a combination of steps including transfusion of RBCs, plasma and platelets in 1:1:1 ratio, preferential use of fresh $\mathrm{RBCs}$, and thawed plasma and concomitant use of coagulation factors and antifibrinolytics $[8,17]$. Such an approach has rekindled interest in 
the administration of FWB to trauma patients with hypovolemia[5]. Subsequently, studies have also analyzed the roles of FWB and BC in patients undergoing cardiovascular, obstetric and oncological surgeries $[5,15,18,19$, 19-21]. Spinal surgery is a rapidly growing specialty, with a humongous $220 \%$ rise in volume of procedures over the past 15 years [1, 3, 4]. With increasing sophistication of technology, spinal surgeries have also grown appallingly in terms of magnitude and complexity. The current prospective RCT was thus planned to compare FWB and CT's effects on the postoperative status and recovery of patients undergoing major SDS. To our knowledge, this is the first study to be published on this topic.

Procurement and storage of $W B$ and $B C$ : FWB can be stored in room temperature for up to $6 \mathrm{~h}$ (warm) or $48 \mathrm{~h}$ at $4-6{ }^{\circ} \mathrm{C}$ (cold) and delivers all cellular and non-cellular components of blood. WB must ideally be utilized in freshly prepared state at the earliest opportunity after thorough screening for infections. Longer storage of warm WB has been associated with complications like bacterial contamination, loss of 2,3-diphosphoglycerate (DPG) and coagulation factors and immunomodulatory effects due to released leucocytic cytokines [22]. In our study, the transfusion of FWB was performed in all patients within $24 \mathrm{~h}$ of procurement. The individual components are separated by centrifugation. The shelf-lives of packed RBCs, FFP and platelets are 21 to 49 days, 36 months $\left(\leq-30{ }^{\circ} \mathrm{C}\right)$ and 5 to 7 days $\left(2{ }^{\circ} \mathrm{C}\right)$, respectively [15].

Storage lesion of RBCs: "RBC storage lesion" describes the complex phenomenon including mechanical and biochemical intraerythrocytic changes which reduce its functional abilities and integrity [23]. These changes include depletion of adenosine 5'-triphosphate (ATP), nitric oxide (NO) and DPG, acidosis, raised intracellular potassium, lipid peroxidation, oxidative injury, alteration of cytoskeleton and apoptosis [24]. These changes augment RBC fragility and lead to hemolysis [25]. Studies have demonstrated the deleterious effects of hemolysis as an essential contributor to mortality in sepsis [26]. SIRS in critically-ill patients may also enhance their susceptibility to transfusion-related complications, especially following transfusion of stored BC [26].

Blood losses in major SDS: Massive BL has been described as loss of one blood volume of patient's total blood $(60 \mathrm{~mL} / \mathrm{kg}$ in adults $) \leq 24 \mathrm{~h}[17,27,28]$. Although the magnitude of BL is significantly high in major SDSs (650 to $2839 \mathrm{ml}$ ), there is no clear definition of "significant BL" in this context [4]. The mean IOBL in our cohort was $888 \mathrm{ml}$. The main goal in such surgeries is to minimize the BL through multipronged strategies like excellent surgical technique, cell saver (CS), antifibrinolytic agents (aprotinin, tranexamic acid, aminocaproic acid), recombinant factor VIIa, platelet cell growth factor, normovolemic, hemodilutional, hypotensive anesthesia and staging of procedures $[4$,
29, 30]. Despite such measures, studies have demonstrated 50 to $81 \%$ transfusion rates following major SDS [4]. The mean intraoperative transfusions in our study were 1.90 and 1.65 units, in FWBG and CG, respectively.

Major observations in our study: All the preoperative demographic and deformity-related characteristics of the two groups were similar. The amount of transfusion received and other intraoperative parameters were also similar. The most significant observations of this study included a statistically significant reduction in the duration of supplemental oxygen dependence and duration of HDU stay in FWBG, as compared with CG. ABGA also showed more acidotic mean arterial $p H$ in $C G$. These differences could be attributed to better structural and functional abilities of RBC in FWB to deliver oxygen to tissues. The amount of additives and preservatives is comparatively low in FWB, so RBCs are potentially in their most physiological and optimal capacity [5].

Recent studies have demonstrated a negative correlation between number of packed RBC transfusions and overall outcome in critical ICU patients [31]. Gruenwald [15] demonstrated greater need for inotropic support, and longer ventilation time and hospital stay in neonates undergoing cardiac surgery following CT compared with FWB transfer. Repine [27] and Spinella [8, 17] demonstrated distinct advantages of FWB transfer in military trauma, including significantly better 24-h and 30-day survival. Blackburn [32] also demonstrated a significant dose-response relationship between postoperative, morbid events like infections and prolonged hospital stay and CT.

Another potential advantage of FWB transfer over CT is the relatively lower possibility of coagulopathy and postoperative BL. Studies have shown significant hemostatic benefits of FWB administration in diverse clinical situations including trauma-induced coagulopathy [9], pediatric cardiovascular procedures [19] and obstetric BL [21]. A recent study published in 2020 has discussed the distinct advantages of modified WB [cold-stored low-titer anti-A and anti-B group O whole blood (LTOWB)] in civilian trauma situations including a 53\% reduction in need for transfusion and twofold improvement in 28-day survival, as compared with CT [10]. These findings contrast with observations in our study, where there was relatively higher postoperative drain output in FWBG, although this difference was not statistically significant. Additionally, serum calcium was reduced marginally, although to a statistically significant level $(9.28 \mathrm{mg} / \mathrm{dl}$ vs. $9.68 \mathrm{mg} / \mathrm{dl}$ ) in $C G$ (but well within acceptable limits), which might be explained by calcium losses incurred by stored BC [5].

Inflammation and transfusion: Another major criticism against CT is the significantly greater possibility of immunological reactions. This phenomenon may be secondary to the excessive release of pro-inflammatory and procoagulant phospholipids from stored RBCs undergoing hemolysis into circulation $[24,33]$. Even in our study, we could observe a 
significant association of raised lactate and IL-6 in patients who underwent $C T$. We also observed a significantly higher occurrence of postoperative facial puffiness (which is a recognized systemic manifestation of immune-mediated reactions) in patients who underwent $C T$. Expectantly; the individuals with facial puffiness also demonstrated elevated IL-6. There was no significant difference in hemoglobin, platelet, electrolytes and coagulation profile between the groups.

IL-6 is a major cytokine released into peripheral circulation after major surgeries, with both pro-inflammatory and anti-inflammatory properties. Persistently raised IL-6 even beyond the initial 5 days is an essential predictor for systemic inflammatory response syndrome (SIRS) and postoperative complications [34, 35]. Previous studies [34, 35] have demonstrated that serum IL-6,10 increase significantly in proportion with the number of stored, non-leucodepleted packed RBC transfusions in patients undergoing thoracic and abdominal surgeries; and these cytokines especially $\mathrm{IL}-10$ is purported to be responsible for immunomodulatory effects of CT. The administration of freshly procured WB demonstrated a reduction in these inflammatory parameters postoperatively, as compared with stored CT.

Some studies $[8,18]$ have demonstrated high rates of immune-mediated acute kidney injury (AKI) and adult respiratory distress syndrome (ARDS) following transfusion of non-leucodepleted FWB transfusion. These reactions to FWB were related to inflammation caused by transfused leucocytes, leading to microvascular endothelial and subsequent end-organ damage. The use of leuco-reduced WB for transfusion can obviate these potential complications [12]. We used leuco-reduced WB in the current study and did not observe such serious complications, including deep infection in both groups.

Most of the evidence on WB transfusion in the literature is based on our experience with military or civilian trauma situations, pediatric cardiovascular surgeries and obstetric bleeding; and predominantly involve young patients. The sample population in our study also involves pediatric and adolescents patients. One of the major reasons for paucity of information on WB transfusions is its lack of availability, especially in North America due to FDA-imposed restrictions $[5,10,16]$. In view of these aforementioned benefits like reduced HDU stay and mitigated postoperative inflammatory response, it may be worthwhile to evaluate whether the addition of FWB transfusion perioperatively in patients undergoing SDS can be a useful addition to Enhanced recovery after surgery (ERAS) program bundles. Nevertheless, for practical reasons previously mentioned, we do not practice early discharge in our patients and such an assessment is beyond the scope of this study.

Limitations of the study: All the data in the current study were obtained from a single center. The sample size is relatively small. Nevertheless, it was a prospective RCT and to our knowledge, there is no other study on FWB transfusion involving patients undergoing spine surgery.

\section{Conclusion}

FWB transfusion can potentially improve the immediate postoperative outcome in patients undergoing major SDS by reducing the duration of stay in intensive care unit and oxygen dependence. The other potential benefits of this practice, based on our study include reduced inflammatory response (reduced lactate and IL-6) and postoperative facial puffiness. However, importance of further validation studies in future to determine precisely the role and safety of FWB in spine surgeries, especially in elderly patients, cannot be understated.

\section{Acknowledgements Nil}

Funding Ganga Orthopedic Research and Education Fund (GOREF).

\section{Declarations}

Conflicts of interest The authors have no conflicts of interest to disclose.

\section{References}

1. Sethi RK, Pong RP, Leveque J-C et al (2014) The Seattle Spine Team approach to adult deformity surgery: a systems-based approach to perioperative care and subsequent reduction in perioperative complication rates. Spine Deform 2:95-103. https://doi. org/10.1016/j.jspd.2013.12.002

2. Sihler KC, Napolitano LM CHEST Postgraduate Education Corner Contemporary reviews in critical care medicine

3. Willner D, Spennati V, Stohl S et al (2016) Spine surgery and blood loss: systematic review of clinical evidence. Anesth Analg 123:1307-1315. https://doi.org/10.1213/ANE.0000000000001485

4. Elgafy H, Bransford RJ, McGuire RA et al (2010) Blood loss in major spine surgery: are there effective measures to decrease massive hemorrhage in major spine fusion surgery? Spine 35:S47-56. https://doi.org/10.1097/BRS.0b013e3181d833f6

5. Pivalizza EG, Stephens CT, Sridhar S et al (2018) Whole blood for resuscitation in adult civilian trauma in 2017: a narrative review. Anesth Analg 127:157-162. https://doi.org/10.1213/ANE.00000 00000003427

6. Pinheiro MK, Tamagne M, Elayeb R et al (2020) Blood microparticles are a component of immune modulation in red blood cell transfusion. Eur J Immunol. https://doi.org/10.1002/eji.20194 8481

7. Cotton BA, Reddy N, Hatch QM et al (2011) Damage control resuscitation is associated with a reduction in resuscitation volumes and improvement in survival in 390 damage control laparotomy patients. Ann Surg 254:598-605. https://doi.org/10.1097/ SLA.0b013e318230089e

8. Spinella PC, Perkins JG, Grathwohl KW et al (2009) Warm fresh whole blood is independently associated with improved survival 
for patients with combat-related traumatic injuries. J Trauma 66:S69-76. https://doi.org/10.1097/TA.0b013e31819d85fb

9. Avery P, Morton S, Tucker H et al (2020) Whole blood transfusion versus component therapy in adult trauma patients with acute major haemorrhage. Emerg Med J EMJ. https://doi.org/10.1136/ emermed-2019-209040

10. Leeper CM, Yazer MH, Neal MD (2020) Whole-blood resuscitation of injured patients: innovating from the past. JAMA Surg. https://doi.org/10.1001/jamasurg.2020.0811

11. Yang J, Skaggs DL, Chan P et al (2018) Raising mean arterial pressure alone restores $20 \%$ of intraoperative neuromonitoring losses. Spine 43:890-894. https://doi.org/10.1097/BRS.00000 00000002461

12. Blajchman MA (2006) The clinical benefits of the leukoreduction of blood products. J Trauma 60:S83-90. https://doi.org/10.1097/ 01.ta.0000199537.09201.7b

13. Villafuerte-Nuñez AE, Téllez-Anguiano AC, Hernández-Díaz O et al (2013) Facial Edema Evaluation Using Digital Image Processing. In: Discrete Dyn. Nat. Soc. https://www.hindawi.com/ journals/ddns/2013/927843/. Accessed 17 May 2020

14. Likert_1932.pdf

15. Gruenwald CE, McCrindle BW, Crawford-Lean L et al (2008) Reconstituted fresh whole blood improves clinical outcomes compared with stored component blood therapy for neonates undergoing cardiopulmonary bypass for cardiac surgery: a randomized controlled trial. J Thorac Cardiovasc Surg 136:1442-1449. https:// doi.org/10.1016/j.jtcvs.2008.08.044

16. Williams J, Merutka N, Meyer D et al (2020) Safety profile and impact of low-titer group $\mathrm{O}$ whole blood for emergency use in trauma. J Trauma Acute Care Surg 88:87-93. https://doi.org/10. 1097/TA.0000000000002498

17. Spinella PC, Holcomb JB (2009) Resuscitation and transfusion principles for traumatic hemorrhagic shock. Blood Rev 23:231240. https://doi.org/10.1016/j.blre.2009.07.003

18. Perkins JG, Cap AP, Spinella PC et al (2011) Comparison of platelet transfusion as fresh whole blood versus apheresis platelets for massively transfused combat trauma patients (CME). Transfus (Paris) 51:242-252. https://doi.org/10.1111/j.1537-2995.2010. 02818.x

19. Manno CS, Hedberg KW, Kim HC et al (1991) Comparison of the hemostatic effects of fresh whole blood, stored whole blood, and components after open heart surgery in children. Blood 77:930-936

20. Mou SS, Giroir BP, Molitor-Kirsch EA et al (2004) Fresh whole blood versus reconstituted blood for pump priming in heart surgery in infants. N Engl J Med 351:1635-1644. https://doi.org/10. 1056/NEJMoa041065

21. Kohli N, Bhaumik S, Jagadesh S et al (2019) Packed red cells versus whole blood transfusion for severe paediatric anaemia, pregnancy-related anaemia and obstetric bleeding: an analysis of clinical practice guidelines from sub-Saharan Africa and evidence underpinning recommendations. Trop Med Int Health TM IH 24:11-22. https://doi.org/10.1111/tmi.13173
22. Hughes JD, Macdonald VW, Hess JR (2007) Warm storage of whole blood for 72 hours. Transfus (Paris) 47:2050-2056. https:// doi.org/10.1111/j.1537-2995.2007.01429.x

23. Zimrin AB, Hess JR (2009) Current issues relating to the transfusion of stored red blood cells. Vox Sang 96:93-103. https://doi. org/10.1111/j.1423-0410.2008.01117.x

24. Hess JR (2010) Red cell storage. J Proteomics 73:368-373. https:// doi.org/10.1016/j.jprot.2009.11.005

25. Kim-Shapiro DB, Lee J, Gladwin MT (2011) Storage Lesion. Role Red Cell Breakdown Transfus (Paris) 51:844-851. https://doi.org/ 10.1111/j.1537-2995.2011.03100.x

26. Larsen R, Gozzelino R, Jeney V et al (2010) A central role for free heme in the pathogenesis of severe sepsis. Sci Transl Med. https:// doi.org/10.1126/scitranslmed.3001118

27. Repine TB, Perkins JG, Kauvar DS, Blackborne L (2006) The use of fresh whole blood in massive transfusion. J Trauma 60:S59-69. https://doi.org/10.1097/01.ta.0000219013.64168.b2

28. Murdock AD, Berséus O, Hervig T et al (2014) Whole blood: the future of traumatic hemorrhagic shock resuscitation. Shock Augusta Ga 41(Suppl 1):62-69. https://doi.org/10.1097/SHK. 0000000000000134

29. Castro FP (2004) Role of activated growth factors in lumbar spinal fusions. J Spinal Disord Tech 17:380-384. https://doi.org/10. 1097/01.bsd.0000110342.54707.19

30. Siska PA, Westrick ER et al (2008) Efficacy of intraoperative cell saver in decreasing postoperative blood transfusions in instrumented posterior lumbar fusion patients. Spine 33:571-575. https://doi.org/10.1097/BRS.0b013e3181657cc1

31. Hébert PC, Wells G, Blajchman MA et al (1999) A multicenter, randomized, controlled clinical trial of transfusion requirements in critical care. Transfusion requirements in critical care investigators, Canadian critical care trials group. N Engl J Med 340:409417. https://doi.org/10.1056/NEJM199902113400601

32. Blackburn CW, Morrow KL, Tanenbaum JE et al (2019) Clinical outcomes associated with allogeneic red blood cell transfusions in spinal surgery: a systematic review. Glob Spine J 9:434-445. https://doi.org/10.1177/2192568218769604

33. Hess JR (2010) Red cell changes during storage. Transfus Apher Sci Off J World Apher Assoc Off J Eur Soc Haemapheresis 43:5159. https://doi.org/10.1016/j.transci.2010.05.009

34. Das BC, Alam SMK, Nashid ZF et al (2017) Change in serum interleukin-6 levels in patients after pancreatoduodenectomy for periampullary cancer. Bangabandhu Sheikh Mujib Med Univ J 10:11-15. https://doi.org/10.3329/bsmmuj.v10i1.31311

35. Fink-Neuboeck N, Lindenmann J, Bajric S et al (2016) Clinical impact of interleukin 6 as a predictive biomarker in the early diagnosis of postoperative systemic inflammatory response syndrome after major thoracic surgery: a prospective clinical trial. Surgery 160:443-453. https://doi.org/10.1016/j.surg.2016.04.004

Publisher's Note Springer Nature remains neutral with regard to jurisdictional claims in published maps and institutional affiliations. 